\title{
II progresso umano: quando la scienza non accresce la conoscenza. Il caso delle cellule STAP
}

\section{Duccio Lombardi}

Dottorando in Area del Farmaco e Trattamenti Innovativi, Dipartimento di Medicina Sperimentale e Clinica, Centro d'Eccellenza DENOTHE, Università degli Studi di Firenze, Firenze

\begin{abstract}
When science does not improve human knowledge and progress: the STAP cells' case
Over the past decade, an increasing number of flaws and mistakes, more or less unintentionally made, were discovered in the biomedical literature. This can undermine the progress of the medical field by allowing the creation of fallacious, or not entirely correct, ideas that may affect not only the research field but, potentially, also the clinical one. In this context, an increasing criticism is targeting the major biomedical journals as well as the peer review process. The latter will almost certainly need to be modified and revised to ensure the integrity of the medical knowledge. Moreover, this topic has recently reached the public opinion thanks to an article deliberately altered and published in Nature; this allows analyzing the main weaknesses of the scientific publication process.
\end{abstract}

Keywords: Cell fate reprogramming, Research ethic, Revision process weaknesses, Misleading science conduct

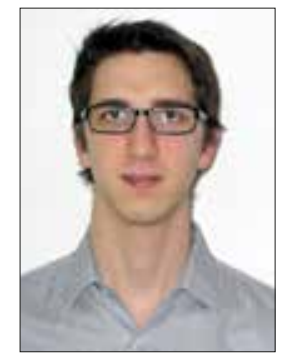

Duccio Lombardi
Quando a fine Gennaio 2014 uscirono su Nature gli articoli "Stimulus-triggered fate conversion of somatic cells into pluripotency" (1) e "Bidirectional developmental potential in reprogrammed cells with acquired pluripotency" (2) inizialmente si generò molto scalpore e interesse all'interno della comunità scientifica. Negli articoli citati si introduceva, infatti, un nuovo metodo che sembrava permettere la conversione di cellule terminalmente differenziate, in questo caso linfociti splenici, in cellule con un fenotipo embrionale, ossia capaci di differenziare in qualsiasi tipo cellulare sotto appropriato stimolo. Nondimeno questa rimodulazione fenotipica e ge-

\section{Accepted: January 26, 2015}

Published online: February 6, 2015

Indirizzo per la corrispondenza:

Dr. Duccio Lombardi

Centro DENOTHE

Università degli Studi di Firenze

V.le G. Pieraccini 6

50139 Firenze

lomb.duccio@gmail.com notipica, che prende il nome di "fate reprogramming", era già stata messa a punto, pubblicata (3) e brevettata sette anni prima dal gruppo di Shinya Yamanaka. Questa scoperta, al momento delle due pubblicazioni, era già valsa a Yamanaka il premio Nobel per la medicina nel 2012. La differenza era che stavolta lo stesso processo era stato effettuato senza però introdurre alcuna modifica diretta nel genoma della cellula. Infatti, nello studio di Yamanaka la riprogrammazione era ottenuta impiegando vettori retrovirali al fine di inserire $i$ quattro geni Oct3/4, Sox2, Klf4, e c-Myc (3). Sebbene quindi la scoperta del "fate reprogramming" non fosse nuova, ciò che maggiormente attraeva era l'innovazione della metodologia. Non si doveva più ricorrere a modifiche del patrimonio genetico della cellula con geni capaci di indurre una trasformazione in senso neoplastico, né lo si doveva fare usando retrovirus, anch'essi capaci di indurre uno switch in senso tumorale. Così facendo si eliminava il rischio di trasformazione maligna, mantenendo però il principale vantaggio legato alla riprogrammazione cellulare: essendo il donatore lo stesso paziente, il rigetto non è un rischio contemplato.

Gli Autori riportavano infatti che, grazie alla metodica STAP (stimulus-triggered acquisition of pluripotency), cellule adulte erano state riprogrammate verso un fenotipo pluripotente con il solo aiuto di un forte e transiente stimolo esterno di intensità sub-letale, ossia l'acidità $(1,2)$. Così facendo, Obokata et al. avrebbero generato cellule con la capacità virtuale di differenziare in qualsiasi tipo cellulare che si ritrova in un adulto, come 
dimostrato, oltre che in vitro, anche dalla capacità delle STAP cell di generare embrioni chimerici se iniettate in blastocisti murine. Analogamente, quando questi embrioni erano stati impiantati, ne erano derivati topi chimerici, ossia con metà patrimonio genetico derivante da quello della STAP.

Nonostante sia documentato in vari organi e differenti contesti che un forte stress sia in grado di indurre modifiche sostanziali nel genotipo e nel comportamento di una cellula, facendola ipoteticamente comportare come una cellula staminale (4-7), tale ipotesi non trovava riscontro quando la tecnica STAP provava a essere riprodotta. Infatti, nei mesi successivi alle pubblicazioni di Nature $(1,2)$, da molti laboratori iniziavano a filtrare indiscrezioni che testimoniavano l'incapacità di replicare la metodica in quasi tutti i suoi punti. Per rispondere alle sempre più numerose voci che chiedevano approfondimenti e chiarimenti sul perché la metodica STAP non fosse riproducibile al di fuori del laboratorio che l'aveva messa a punto, la RIKEN, maggiore istituzione di ricerca giapponese e istituzione presso cui erano stati svolti gli studi, avviò un'indagine sui controversi articoli. Fu così che, a due mesi dalla pubblicazione, il RIKEN Center for Developmental Biology di Kobe annunciò che alcune delle immagini riportate negli articoli erano state manipolate. A seguire, nel Luglio del 2014, Nature ritirò i due articoli indicando con precisione quali fossero stati le manipolazioni e i maggiori punti di discrepanza $(8,9)$.

Il 3 Settembre del 2014 fu avviata una seconda indagine ad opera di un team di 7 scienziati, non impiegati alla RIKEN, il cui scopo era quello di indagare più a fondo sulle linee cellulari incriminate e sui campioni tissutali analizzati, così da capirne l'origine, le caratteristiche e quali effettive modifiche avessero subito. La commissione di scienziati prese in esame campioni di DNA da STAP cell, cellule staminali embrionali (ESC) impiegate nello studio come campione di controllo, e da topi generati per impianto di embrioni chimerici. Furono inoltre analizzati appunti di laboratorio, documenti ed e-mail degli Autori coinvolti, che vennero successivamente interrogati singolarmente.

L'esito conclusivo del lavoro di questa seconda equipe di scienziati fui reso pubblico lo scorso 26 Dicembre. Quanto emerse fu non solo che altre due figure erano state volutamente alterate ma, soprattutto, che alcune colture di STAP cell erano contaminate dalla presenza di 3 differenti linee di ESC, una delle quali era la fonte con cui era stata sviluppata la linea murina chimerica. Nonostante lo studio accurato del materiale preso in analisi, gli "investigatori" dichiararono di non essere in grado di stabilire come la contaminazione potesse essere avvenuta, affermando anche, però, che fosse difficile immaginare una contaminazione accidentale con tre distinte linee di ESC, essendo inoltre il lavoro eseguito da Autori di esperienza e autorevolezza riconosciute a livello globale. Anche se era molto probabile che tale contaminazione non fosse accidentale, la commissione, nell'impossibilità di stabilire chi fosse il responsabile della contaminazione, non ha potuto determinare in maniera conclusiva se si trattasse di un evento fortuito o artificiale.
Nonostante in questo caso la verità sia emersa, anche se non del tutto, permettendo così di sottrarre alla conoscenza di natura biomedica informazioni falsate, alcune domande di fondo permangono. Come è possibile che questi due articoli abbiano passato la fase di revisione e siano stati pubblicati su una rivista del calibro di Nature? Quanti altri articoli sono presenti in letteratura che riportano dati non del tutto corretti o apertamente alterati? E ancora, che impatto hanno questi sull'intera conoscenza biomedica?

Tutto ciò evidenzia quindi qualcosa di già noto: il sistema di revisione di un articolo presenta più punti deboli. In primis, come evidenziato dal caso delle STAP, chi revisiona il lavoro svolto non ha accesso diretto ai campioni e ai dati grezzi delle varie analisi, sebbene questo possa essere in qualsiasi momento richiesto, ma raramente accade. In questo contesto i punti cardine vengono quindi a essere l'onestà intellettuale del ricercatore e la sua etica professionale, che in campo sanitario dovrebbero essere ferree e indubitabili, data l'importanza delle questioni prese in considerazione. Purtroppo però l'etica è e sarà sempre influenzata da morale e visione personale, e contro questa natura umana è difficile combattere, se non mediante la responsabilizzazione degli individui sin dai primi momenti del loro percorso di formazione professionale.

Allo stesso tempo però va considerato anche il ruolo di chi revisiona il lavoro, che molto spesso è uno scienziato che si occupa della stessa specifica materia e che può avere un interesse personale nel fare in modo che certe pubblicazioni, per quanto esemplari, siano totalmente ostacolate o temporaneamente bloccate. In questo modo chi revisiona il lavoro, generalmente un group leader di chiara influenza scientifica, può fare in modo che eventuali propri dati analoghi siano pubblicati prima rispetto a quelli dell'articolo che è chiamato a referare. Altresì, un lavoro potrebbe essere favorito senza grosse indagini semplicemente perché in sintonia con le tesi di chi revisiona o perché in linea con i lavori che costui ha già pubblicato o ha intenzione di pubblicare in un prossimo futuro. Anche in questo caso, non diversamente da prima, è l'etica che dovrebbe guidare l'azione del singolo a essere sotto processo, ed è proprio tale etica che può essere messa da parte a discrezione del soggetto. Per questo motivo i lavori sono revisionati sempre da più di un esperto del campo, anche se non è inusuale che tra scienziati della stessa area possano crearsi sorte di cordate volte a far prevalere un concetto piuttosto che un altro, sia perché costoro condividono la stessa idea sia perché spesso cooperano tra loro formalmente o meno.

Infine va considerata quella che è l'effettiva riproduzione, in laboratori differenti da quello di origine, di metodiche e tecniche illustrate nei vari lavori. II numero di paper che sono effettivamente riprodotti in toto o parzialmente è molto basso e spesso, quando sono riprodotti, non danno alcun esito o almeno non quello atteso. Nel 2005 John loannidis, professore di medicina presso la Stanford University, scrisse un lavoro che ebbe grande risonanza all'interno della comunità scientifica. Nell'articolo egli dimostrava, tramite una 
meta-analisi di 45 tra gli articoli biomedici più citati dell'epoca, che circa il $50 \%$ dei suddetti lavori risultava smentito da studi successivi o che questi si erano semplicemente rivelati mendaci per manipolazione dei dati o campioni troppo piccoli per essere ritenuti statisticamente significativi (10). Sebbene tale percentuale sia stata rivista da studi successivi intorno al $20 \%$, nondimeno la gravità del problema permane.

Tutto ciò è presumibilmente dovuto all'estrema competizione che si ha oggi in campo biomedico. In una situazione del genere l'imperativo diventa "pubblicare o morire": per progredire nella propria carriera spesso il ricercatore necessita di un numero minimo di pubblicazioni su riviste ad alto impact factor, e ciò influisce ovviamente sulla qualità della ricerca, la quale passa in secondo piano rispetto all'etica professionale. Analogamente, qualora le riviste con maggiore influenza scientifica prediligano la pubblicazione di determinati argomenti, generalmente i cosiddetti "argomenti caldi del momento", lo scienziato pur di accedervi può essere tentato dal mettere da parte l'etica professionale. In questo caso la rivista, oltre all'individuo che sottomette la propria etica alla logica della "grande pubblicazione", è responsabile di un notevole danno al progresso della scienza. Infatti, favorendo argomenti più "trendy", la rivista spinge indirettamente sempre più scienziati a cambiare il proprio campo di interesse e a fare di tutto pur di raggiungere l'obiettivo di pubblicare in modo prestigioso. Quel che però è ancora peggio è che, così facendo, le riviste scientifiche si rendono colpevoli di una progressione asincrona delle varie sotto-discipline biomediche, come sostenuto dal premio Nobel per la medicina del 2013 Randy Sheckman, che dal podio di Stoccolma annunciò il boicottaggio di Nature, Science e Cell. II premio Nobel, al pari del Nobel per la fisica Peter Higgs, ritiene infatti che tali riviste danneggino la scienza pubblicando in base all'appeal mediatico di uno studio, piuttosto che alla sua reale rilevanza scientifica.

Ovviamente, tutto ciò sta mettendo seriamente sotto processo il meccanismo della peer review, che troppo spesso risulta inadeguato per gli scopi prefissi: controllare la qualità della scienza che ogni giorno è pubblicata. In tale ottica, la soluzione che al momento sembra migliore è quella della open science, la quale obbliga a rendere pubblico ogni dettaglio sperimentale, facendo sì che, oltre al controllo operato dai revisori, si abbia anche un controllo di qualità postumo rispetto alla pubblicazione. Se infatti un articolo è più facilmente accessibile, un maggior numero di scienziati non solo vi poserà gli occhi, ma ne trarrà spunto nel mettere a punto i propri esperimenti, eseguendo in questo modo una riproduzione indiretta dell'esperimento.

Solo in questo modo si favoriscono quindi la scienza, la medicina, la cura e, soprattutto, il paziente.

\section{Disclosures}

Financial support: No financial support was received for this submission.

Conflict of interest: The author has no conflict of interest with this submission.

\section{Bibliografia}

1. Obokata H, Wakayama T, Sasai Y, et al. Stimulus-triggered fate conversion of somatic cells into pluripotency. Nature 2014;505 (7485):641-7. Retracted.

2. Obokata H, Sasai $\mathrm{Y}$, Niwa $\mathrm{H}$, et al. Bidirectional developmental potential in reprogrammed cells with acquired pluripotency. Nature 2014;505(7485):676-80. Retracted.

3. Takahashi K, Tanabe K, Ohnuki M, et al. Induction of pluripotent stem cells from adult human fibroblasts by defined factors. Cell 2007;131(5):861-72.

4. Mody N, Parhami F, Sarafian TA, Demer LL. Oxidative stress modulates osteoblastic differentiation of vascular and bone cells. Free Radic Biol Med 2001;31(4):509-19.

5. Kusaba T, Lalli M, Kramann R, Kobayashi A, Humphreys BD. Differentiated kidney epithelial cells repair injured proximal tubule. Proc Natl Acad Sci U S A 2014;111(4):1527-32.

6. Tsai MC, Chen L, Zhou J, et al. Shear stress induces syntheticto-contractile phenotypic modulation in smooth muscle cells via peroxisome proliferator-activated receptor alpha/delta activations by prostacyclin released by sheared endothelial cells. Circ Res 2009;105(5):471-80.

7. King MR, Ismail AS, Davis LS, Karp DR. Oxidative stress promotes polarization of human T cell differentiation toward a T helper 2 phenotype. J Immunol 2006;176(5):2765-72.

8. Obokata H, Wakayama T, Sasai Y, et al. Retraction: Stimulustriggered fate conversion of somatic cells into pluripotency. Nature 2014;511(7507):112.

9. Obokata H, Sasai $\mathrm{Y}$, Niwa $\mathrm{H}$, et al. Retraction: Bidirectional developmental potential in reprogrammed cells with acquired pluripotency. Nature 2014;511(7507):112.

10. Ioannidis JP. Why Most Published Research Findings Are False. PLoS Med 2005;2(8):e124. 